\title{
Gene expression profiling of CD133-positive cells in coronary artery disease
}

\author{
JIAYU LI ${ }^{1}$, CHANGYU ZHOU ${ }^{2}$, JIARUI LI ${ }^{3}$, YINGCHUN WAN ${ }^{4}$, TAO LI $^{5}$, \\ PIYONG $\mathrm{MA}^{6}$, YINGJIAN WANG ${ }^{7}$ and HAIYAN SANG ${ }^{1}$ \\ Departments of ${ }^{1}$ Cardiology and ${ }^{2}$ Gastroenterology, China-Japan Union Hospital of Jilin University; \\ ${ }^{3}$ Department of Pharmacy, The Tumor Hospital of Jilin; Departments of ${ }^{4}$ Endocrinology, ${ }^{5}$ Anesthesiology, \\ ${ }^{6}$ Emergency and ${ }^{7}$ Gynaecology, China-Japan Union Hospital of Jilin University, \\ Changchun, Jilin 130033, P.R. China
}

Received October 30, 2014; Accepted June 26, 2015

DOI: $10.3892 / \mathrm{mmr} .2015 .4359$

\begin{abstract}
Gene expression profiles of CD133-positive cells from patients with coronary artery disease (CAD) were analyzed to identify key genes associated with cardiac therapy. Furthermore, the effect of exercise on gene expression was also investigated. Gene expression data set (accession number: GSE18608) was downloaded from the Gene Expression Omnibus, including blood samples from four healthy subjects $(\mathrm{H})$, and from 10 patients with coronary artery disease at baseline (B) and after 3 months (3M) of exercise. Differential analysis was performed for $\mathrm{H}$ vs. $\mathrm{B}$ and $\mathrm{H}$ vs. $3 \mathrm{M}$ using limma package of R. Two-way cluster analysis was performed using the expression levels of the differentially expressed genes (DEGs) by package pheatmap of $R$. Functional enrichment analysis was applied on the DEGs using the Database for Annotation, Visualization and Integrated Discovery. Relevant small molecules were predicted using the Connectivity map database (cMap). A total of 131 and 71 DEGs were identified in patients with CAD prior to and following 3 months of exercise. The two groups of DEGs were compared and 44 genes overlapped. In cluster analysis with the expression levels of the common DEGs, patients with CAD could be well separated from the healthy controls. Functional enrichment analysis showed that response to peptide hormone stimulus and anti-apoptosis pathways were significantly enriched in the common DEGs. A total of 12 relevant small molecules were revealed by cMap based upon the expression levels of common DEGs, such as 5252917 and MG-262. Three months of exercise in part normalized the gene expression in CAD patients.
\end{abstract}

Correspondence to: Dr Changyu Zhou, Department of Gastroenterology, China-Japan Union Hospital of Jilin University, 126 Xiantai Street, Changchun, Jilin 130033, P.R. China E-mail: longyuuz@163.com

Key words: coronary artery disease, gene expression profiles, differentially expressed genes, functional enrichment analysis, small molecules
The genes not altered by exercise may be the targets of small molecules, such as 5252917 and MG-262.

\section{Introduction}

Coronary artery disease (CAD) is the most common type of heart disease and cause of heart attacks. Despite the improvement of pharmacological therapy and coronary revascularization procedures, there remains a requirement for novel therapeutic approaches $(1,2)$. Stimulation of vascular and cardiac repair mechanisms, such as those mediated by stem/progenitor cells, has become a focus of cardiovascular research (3).

Experimental and clinical studies have suggested the feasibility and safety of cell-based therapies in patients with ischemic cardiomyopathy $(4,5)$. To date, different autologous adult stem and progenitor cells, in particular several subtypes of bone marrow-derived cells, isolated adipose tissue-derived and cardiac-derived stem/progenitor cells have gained attention from researchers. CD133-positive cells have shown the ability to home to injured myocardium (6) and promote cardiac recovery (7-9). The molecular mechanisms underlying the cardiac repair process mediated by CD133-positive cells have also been investigated. Ahmadi et al (8) suggested that they may induce myogenesis as well as angiogenesis. Bonanno et al (10) reported that CD133-positive cells differentiate into endothelial- and cardiomyocyte-like cells in vitro. However, several studies have reported that the numbers of bone marrow-derived progenitor cells with endothelial differentiation potential are reduced in patients with CAD $(11,12)$. Therefore, it is necessary to determine the impact of CAD on the gene expression of CD133-positive cells, which may advance the understanding regarding the potential therapeutic mechanism of CD133-positive cells.

Although Liu et al (13) determined certain alterations in the gene expression of CD133-positive progenitor cells from CAD patients, the present study aimed to obtain more information via currently available bioinformatic tools, such as cluster analysis and functional enrichment analysis. Relevant small molecules were also predicted, which could provide clues for future drug development. In addition, the effect of 


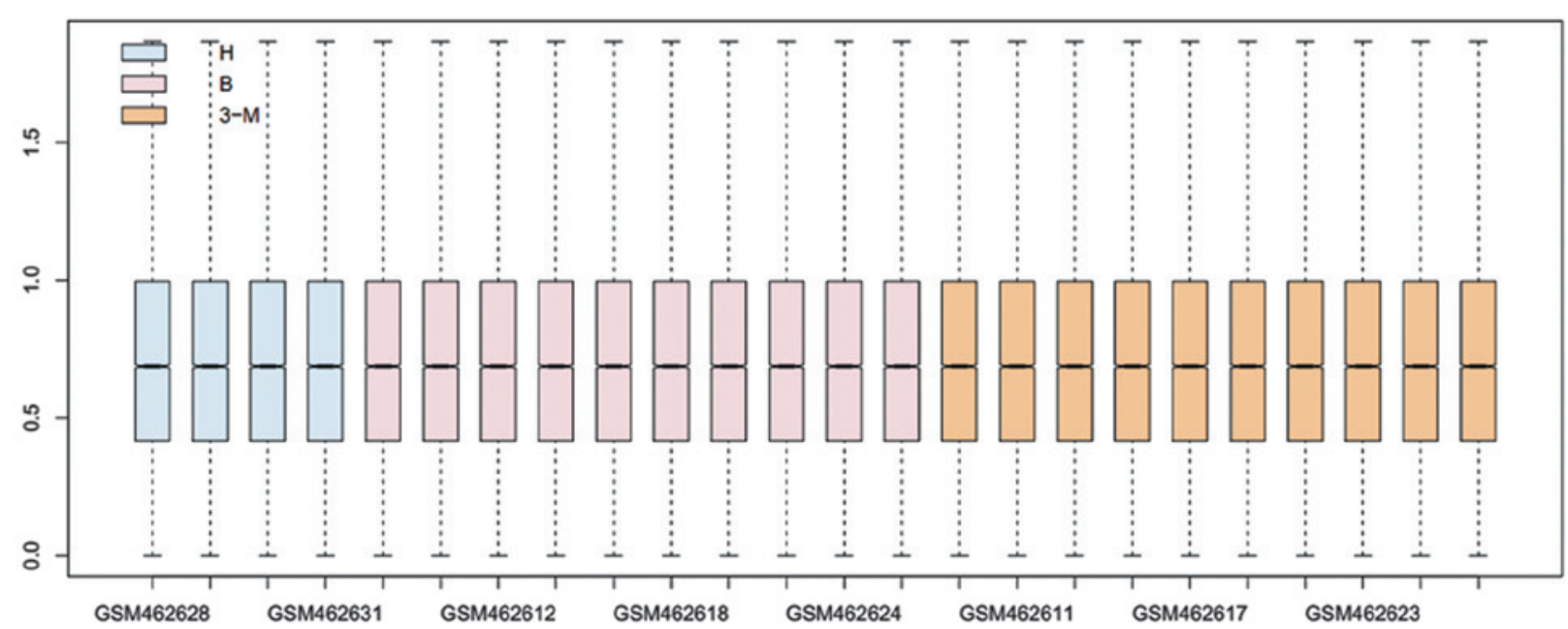

Figure 1. Box plots for normalized gene expression data. Healthy $(\mathrm{H})$ samples are in blue, while coronary artery disease samples prior to (B) and following 3 months of exercise (3-M) are shown in pink and orange, respectively. Black lines in the boxes represent medians.

exercise on the gene expression of CD133-positive cells was also investigated.

\section{Materials and methods}

Gene expression data. Gene expression data set (accession number GSE18608) (13) was downloaded from public Gene Expression Omnibus database (GEO, http://www.ncbi.nlm. nih.gov/geo/). This dataset included blood samples from four healthy subjects $(\mathrm{H})$, and from 10 patients with coronary artery disease at baseline (B) and after 3 months of exercise (3M), which was used for isolation of CD133-positive cells. Gene expression levels were measured using GPL570 [HG-U133_Plus_2] Affymetrix Human Genome U133 Plus 2.0 Array (Affymetrix Inc., Santa Clara, CA, USA). Probe annotations were also acquired.

Pre-treatment and differential analysis. The 54,675 probes were all mapped to genes. A $\log 2$ transformation was applied on the gene expression levels (14). Differential analysis was performed for $\mathrm{H}$ vs. B and $\mathrm{H}$ vs. 3M using limma (Linear Models for Microarray Analysis) (15) package of Bioconductor R (available at http://www.bioconductor.org/packages/release/ bioc/html/ limma.html). Multiple testing correction using the Benjamini-Hochberg method (16) was performed to adjust the P-value to the false discovery rate (FDR). FDR $<0.25$ was set as the cut-off to screen for the significant DEGs.

DEGs of $\mathrm{H}$ vs. B were compared with those of $\mathrm{H}$ vs. $3 \mathrm{M}$ to screen out unique genes differentially expressed prior to and following exercise. In addition, Student's t-test was performed for unique DEGs between the groups. $\mathrm{P}<0.05$ was set as the cut-off.

Clusteranalysis. Two-way cluster analysis was performed using the expression levels of the DEGs by package pheatmap (17) in statistical software $\mathrm{R}$ ( $\mathrm{R}$ version, 2.15 , pheatmap version, 0.6.1; R Core Team, Vienna, Austria). Euclidean distance was adopted to cluster the genes and produce the dendrograms.
Functional enrichment analysis. Functional enrichment analysis was performed for the DEGs using Database for Annotation, Visualization and Integration Discovery (DAVID, http://david.abcc.ncifcrf.gov/) (18) online tools. The statistical method was performed based upon the hypergeometric distribution. $\mathrm{P}<0.05$ was selected as the cut-off.

Prediction of relevant small molecules. Connectivity map database (CMap; http://broad.mit.edu.rpa.skh.org.tw:81/cmap) is designed to link gene patterns associated with disease to corresponding patterns produced by drug candidates $(19,20)$. Relevant small molecules were predicted using the DEGs of $\mathrm{H}$ vs. $3 \mathrm{M}$ and those with Iscorel $>0.8$ were retained.

\section{Results}

Differentially expressed genes. Normalized gene expression data are shown in Fig. 1. A total of 131 and 71 DEGs were identified in patients with CAD prior to and following exercise, respectively. Fewer DEGs were observed in patients after 3 months of exercise. The two groups of DEGs were compared and 44 common genes were identified (Fig. 2A).

A t-test was performed using the expression levels of the DEGs for $\mathrm{H}$ vs. $\mathrm{B}$ and $\mathrm{H}$ vs. $3 \mathrm{M}$ (Fig. 3 and Table I). The difference was significant, which confirmed the reliability of the DEGs.

Cluster analysis result. The cluster analysis result is shown in Fig. 2B. CAD samples could be well separated from healthy controls, confirming the reliability of the DEGs. In addition, CAD samples prior to and following exercise were clustered into one group with expression levels of common DEGs, suggesting that 3 months of exercise would not bring significant improvements in patients and further drug treatment may be required.

Functional enrichment analysis. Functional enrichment analysis showed that response to peptide hormone stimulus was most significantly enriched in the common DEGs (Table II), 

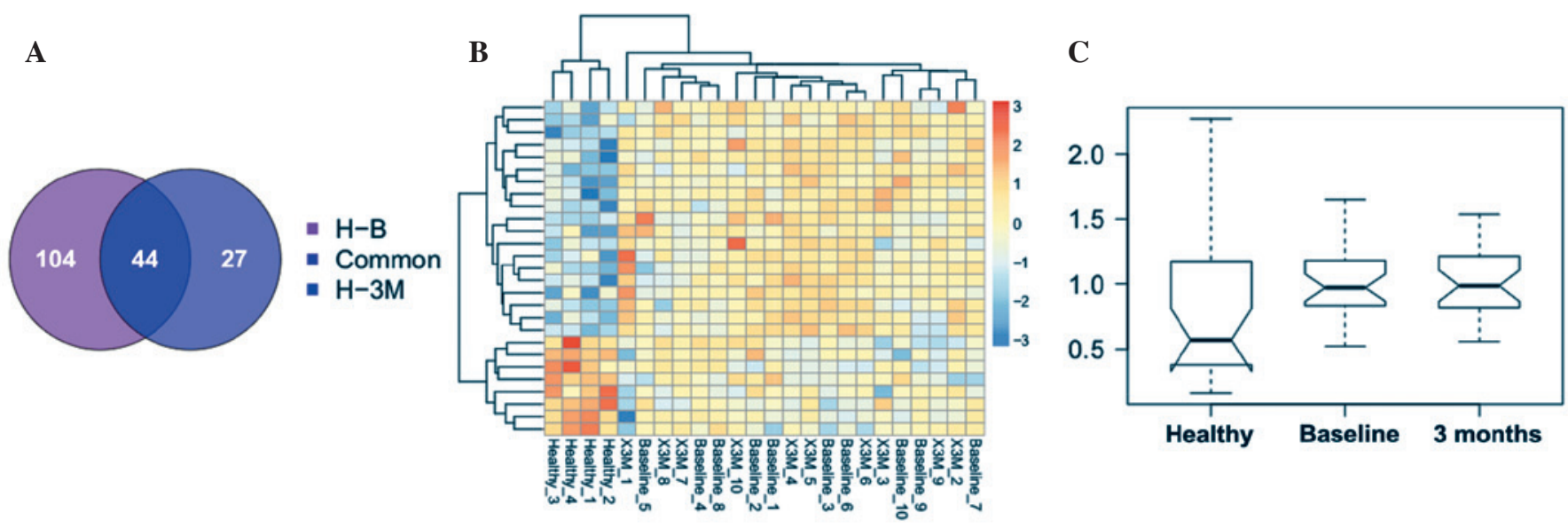

Figure 2. (A) Venn diagram describing the number of differentially expressed genes in patients with coronary artery disease prior to (H-B) and following 3 months of exercise (H-3M). (B) Heatmap demonstrates hierarchical clustering analysis result based on the common differentially expressed genes. (C) Box plot for the expression levels of the common differentially expressed genes among the three groups.

A

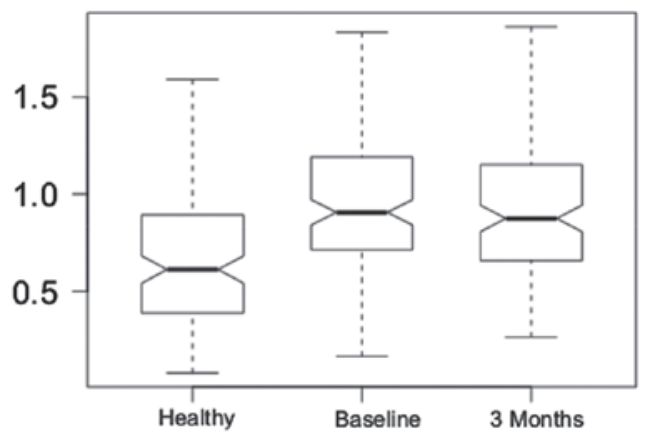

B

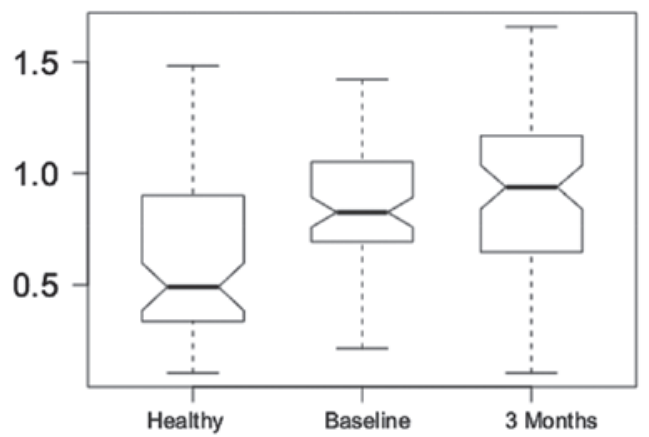

Figure 3. Box plots for expression levels of the unique differentially expressed genes (A) in patients with coronary artery disease prior to exercise and (B) in patients with coronary artery disease after 3 months of exercise.

Table I. Student's t-test result based upon the expression levels of differentially expressed genes.

\begin{tabular}{lccc}
\hline Comparison & Baseline specific & 3M specific & Common \\
\hline H vs. B & $8.82 \mathrm{E}-05$ & $4.81-04$ & $4.21 \mathrm{E}-03$ \\
H vs. 3M & $6.15 \mathrm{E}-04$ & $1.70 \mathrm{E}-04$ & $5.36 \mathrm{E}-02$ \\
\hline
\end{tabular}

$\mathrm{H}$, healthy controls; $\mathrm{B}$, patients with coronary artery disease before exercise; $3 \mathrm{M}$, patients with coronary artery disease after 3 months of exercise.

including protein kinase $\mathrm{C} \alpha$ (PRKCA), protein kinase $\mathrm{C}$ । (PRKCI) and phosphodiesterase 3B (PDE3B). In addition, anti-apoptosis and regulation of glucose transport pathways were also significantly over-represented by the common DEGs.

Relevant small molecules. A total of 12 relevant small molecules were revealed by CMap based upon the expression levels of common DEGs (Table III). 5252917 [corresponding to N-(2-benzooxazol-2-yl-phenyl) -4-methyl-benzenesulfonamide] was the most negatively correlated small molecule.

\section{Discussion}

In the present study, a total of 131 and 71 DEGs were identified in patients with CAD prior to and following 3 months of exercise. The fewer DEGs identified after 3 months of exercise suggested that exercise may normalize the expression of certain genes in CD133-positive cells collected from patients with CAD. However, further comparative study indicated 44 common genes prior to and following 3 months of exercise, illustrating that these genes were not altered by exercise and further treatment for these genes is necessary.

Functional enrichment analysis showed that response to peptide hormone stimulus was the most significant functional term enriched in the common DEGs, including PRKCA, PRKCI, and PDE3B. Protein kinase C (PKC) is a family of serine- and threonine-specific protein kinases that can be activated by calcium and the second messenger diacylglycerol. PKC family members phosphorylate a wide variety of protein targets and are known to be involved in diverse cellular signaling pathways. Braz et al (21) first reported that PRKCA regulates cardiac contractility and propensity towards heart failure. Hambleton et al (22) further indicated that inducible and myocyte-specific inhibition of PRKCA enhances cardiac contractility and protects against infarction-induced heart 
Table II. Functional enrichment analysis result for the common differentially expressed genes.

\begin{tabular}{llll}
\hline Term & Count & P-value & Genes \\
\hline GO:0043434 response to peptide hormone stimulus & 3 & $1.57 \mathrm{E}-02$ & PRKCA, PRKCI, PDE3B \\
GO:0006916 anti-apoptosis & 3 & $2.70 \mathrm{E}-02$ & SERPINB9, PRKCI, POLB \\
GO:0046324 regulation of glucose import & 2 & $4.07 \mathrm{E}-02$ & PRKCA, PRKCI \\
GO:0010827 regulation of glucose transport & 2 & $4.19 \mathrm{E}-02$ & PRKCA, PRKCI \\
GO:0009187 cyclic nucleotide metabolic process & 2 & $4.67 \mathrm{E}-02$ & PDE3B, GUCY1A3 \\
GO:0019899 enzyme binding & 4 & $1.37 \mathrm{E}-02$ & PDE3B, NBEA, POLB, GGA2
\end{tabular}

PRKCA, protein kinase C $\alpha$; PRKCI, protein kinase C 1 ; PDE3B, phosphodiesterase 3B; SERPINB9, Serpin B9.

Table III. Relevant small molecules.

\begin{tabular}{lcr}
\hline CMap name & Enrichment & P-value \\
\hline 5252917 & -0.98 & $6.80 \mathrm{E}-04$ \\
MG-262 & -0.91 & $1.30 \mathrm{E}-03$ \\
DL-thiorphan & -0.90 & $1.91 \mathrm{E}-02$ \\
Biotin & -0.90 & $2.08 \mathrm{E}-03$ \\
CP-944629 & -0.86 & $6.80 \mathrm{E}-04$ \\
AH-6809 & -0.86 & $4.15 \mathrm{E}-02$ \\
5230742 & -0.85 & $4.58 \mathrm{E}-02$ \\
Alsterpaullone & -0.82 & $1.17 \mathrm{E}-02$ \\
PNU-0251126 & -0.80 & $1.40 \mathrm{E}-04$ \\
Alfadolone & 0.82 & $1.20 \mathrm{E}-02$ \\
Ketoconazole & 0.82 & $1.93 \mathrm{E}-03$ \\
5211181 & 0.92 & $1.37 \mathrm{E}-02$ \\
\hline
\end{tabular}

failure. Hambleton (23) drew a similar conclusion. PRKCI is involved in the regulation of myocardial coherence and remodeling during cardiac morphogenesis (24). Phosphodiesterase type 3 (PDE3) is an important regulator of cAMP-mediated responses within the cardiovascular system. Sun et al (25) indicated that PDE3A is the main subtype of PDE3 expressed in platelets and cardiac ventricular myocytes, and is responsible for the functional changes caused by PDE3 inhibition. PDE3B may exhibit a different role in the cardiovascular system, however, further studies are required to determine this. Apoptosis is important in cardiovascular diseases, such as atherosclerosis, ischemic heart disease and congestive heart failure. It was demonstrated that three DEGs were involved in anti-apoptosis pathways: Serpin B9 (SERPINB9), PRKCI and DNA polymerase $\beta$. SERPINB9 is a member of the serpin family that has been found to be an intrinsic inhibitor of granzyme B, which is associated with apoptosis and thus is involved in cardiovascular diseases (26). A protective role of SERPINB9 against apoptosis has been reported in in vivo and in vitro models $(27,28)$. All of these findings suggest that the above genes involved in hormone response and apoptosis may serve as crucial therapeutic targets for CAD.

To further examine the underlying treatment approaches for the 44 genes, which were not altered by exercise, the CMap database was used to predict the small molecules. As a result, 12 small molecules were obtained, such as 5252917 and MG-262. 5252917 was previously described as a mitotic inhibitor and thus induces cell apoptosis (29), and MG-262 is a proteasome inhibitor (30). Zheng et al (31) indicated that pharmacologically induced proteasome inhibition is sufficient to activate autophagy in cardiomyocytes. Stangl et al (32) reported that inhibition of the ubiquitin-proteasome pathway induces differential heat shock protein responses in cardiomyocytes and results in early cardiac protection. These studies suggest that proteasome inhibitors may be used in the treatment of cardiovascular diseases.

In conclusion, a number of DEGs were revealed in CD133-positive cells obtained from patients with CAD. These findings may advance the understanding of the molecular mechanisms underlying cardiac repair and thus benefit cell-based therapy development. Exercise showed a positive effect on the gene expression of CD133-positive cells, but did not completely reverse it. The non-altered genes may require treatment with small molecules drugs..

\section{Acknowledgements}

The authors wish to thank Fenghe (Shanghai) Information Technology Co., Ltd (Shanghai, China) for their ideas and help, which gave a valuable added dimension to our research. 


\section{References}

1. Landmesser U and Drexler H: Chronic heart failure: An overview of conventional treatment versus novel approaches. Nat Clin Pract Cardiovasc Med 2: 628-638, 2005.

2. Ford ES, Ajani UA, Croft JB, Critchley JA, Labarthe DR, Kottke TE, Giles WH and Capewell S: Explaining the decrease in U.S. deaths from coronary disease, 1980-2000. N Engl J Med 356: 2388-2398, 2007.

3. Landmesser U, Wollert KC and Drexler H: Potential novel pharmacological therapies for myocardial remodeling. Cardiovasc Res 81: 519-527, 2009.

4. Schächinger V, Erbs S, Elsässer A, Haberbosch W, Hambrecht R, Hölschermann H, Yu J, Corti R, Mathey DG, Hamm CW, et al: Improved clinical outcome after intracoronary administration of bone-marrow-derived progenitor cells in acute myocardial infarction: Final 1-year results of the REPAIR-AMI trial. Eur Heart J 27: 2775-2783, 2006.

5. Segers VF and Lee RT: Stem-cell therapy for cardiac disease. Nature 451: 937-942, 2008.

6. Schots R, De Keulenaer G, Schoors D, Caveliers V, Dujardin M, Verheye S, Van Camp G, Franken PR, Roland J, Van Riet I and Everaert $\mathrm{H}$ : Evidence that intracoronary-injected $\mathrm{CD} 13+$ peripheral blood progenitor cells home to the myocardium in chronic postinfarction heart failure. Exp Hematol 35: 1884-1890, 2007.

7. Bartunek J, Vanderheyden M, Vandekerckhove B, Mansour S, De Bruyne B, De Bondt P, Van Haute I, Lootens N, Heyndrickx G and Wijns W: Intracoronary injection of CD133-positive enriched bone marrow progenitor cells promotes cardiac recovery after recent myocardial infarction: Feasibility and safety. Circulation 112: I178-I183, 2005.

8. Ahmadi H, Baharvand H, Ashtiani SK, Soleimani M, Sadeghian H, Ardekani JM, Mehrjerdi NZ, Kouhkan A, Namiri M, Madani-Civi M, et al: Safety analysis and improved cardiac function following local autologous transplantation of CD133+ enriched bone marrow cells after myocardial infarction. Curr Neurovasc Res 4: 153-160, 2007.

9. Klein H, Ghodsizad A, Marktanner R, Poll L, Voelkel T, Mohammad Hasani MR, Piechaczek C, Feifel N, Stockschlaeder M, Burchardt ER, et al: Intramyocardial implantation of CD133+ stem cells improved cardiac function without bypass surgery. Heart Surg Forum 10: E66-E69, 2007.

10. Bonanno G, Mariotti A, Procoli A, Corallo M, Rutella S, Pessina G, Scambia G, Mancuso S and Pierelli L: Human cord blood CD133+ cells immunoselected by a clinical-grade apparatus differentiate in vitro into endothelial- and cardiomyocyte-like cells. Transfusion 47: 280-289, 2007.

11. Heeschen C, Lehmann R, Honold J, Assmus B, Aicher A, Walter DH, Martin H, Zeiher AM and Dimmeler S: Profoundly reduced neovascularization capacity of bone marrow mononuclear cells derived from patients with chronic ischemic heart disease. Circulation 109: 1615-1622, 2004.

12. Scheubel RJ, Zorn H, Silber RE, Kuss O, Morawietz H, Holtz J and Simm A: Age-dependent depression in circulating endothelial progenitor cells in patients undergoing coronary artery bypass grafting. J Am Coll Cardiol 42: 2073-2080, 2003.

13. Liu D, Glaser AP, Patibandla S, Blum A, Munson PJ, McCoy JP, Raghavachari N and Cannon RO: Transcriptional profiling of CD133(+) cells in coronary artery disease and effects of exercise on gene expression. Cytotherapy 13: 227-236, 2011.

14. Fujita A, Sato JR, Rodrigues Lde O, Ferreira CE and Sogayar MC: Evaluating different methods of microarray data normalization. BMC Bioinformatics 7: 469, 2006.

15. Smyth GK: Limma: Linear models for microarray data. In: Bioinformatics and computational biology solutions using $\mathrm{R}$ and Bioconductor. Springer, pp397-420, 2005.
16. Benjamini $\mathrm{Y}$ and Hochberg Y: Controlling the false discovery rate: a practical and powerful approach to multiple testing. Journal of the Royal Statistical Society. Series B (Methodological) 289-300, 1995.

17. Kolde R: Pheatmap: Pretty heatmaps. R package version 0.61 : 2012.

18. Huang DW, Sherman BT and Lempicki RA: Systematic and integrative analysis of large gene lists using DAVID bioinformatics resources. Nat Protoc 4: 44-57, 2009.

19. Evan GI and Vousden KH: Proliferation, cell cycle and apoptosis in cancer. Nature 411: 342-348, 2001.

20. Catania A, Urban S, Yan E, Hao C, Barron Gand Allalunis-Turner J: Expression and localization of cyclindependent kinase 5 in apoptotic human glioma cells. Neuro Oncol 3: 89-98, 2001.

21. Braz JC, Gregory K, Pathak A, Zhao W, Sahin B, Klevitsky R, Kimball TF, Lorenz JN, Nairn AC, Liggett SB, et al: PKC-alpha regulates cardiac contractility and propensity toward heart failure. Nat Med 10: 248-254, 2004.

22. Hambleton M, York A, Sargent MA, Kaiser RA, Lorenz JN, Robbins J and Molkentin JD: Inducible and myocyte-specific inhibition of PKCalpha enhances cardiac contractility and protects against infarction-induced heart failure. Am J Physiol Heart Circ Physiol 293: H3768-H3771, 2007.

23. Hambleton M, Hahn H, Pleger ST, Kuhn MC, Klevitsky R, Carr AN, Kimball TF, Hewett TE, Dorn GW 2nd, Koch WJ and Molkentin JD: Pharmacological- and gene therapy-based inhibition of protein kinase Calpha/beta enhances cardiac contractility and attenuates heart failure. Circulation 114: 574-582, 2006.

24. Rohr S, Bit-Avragim N and Abdelilah-Seyfried S: Heart and soul/PRKCi and nagie oko/Mpp5 regulate myocardial coherence and remodeling during cardiac morphogenesis. Development 133: 107-115, 2006.

25. Sun B, Li H, Shakur Y, Hensley J, Hockman S, Kambayashi J, Manganiello VC and Liu Y: Role of phosphodiesterase type $3 \mathrm{~A}$ and $3 \mathrm{~B}$ in regulating platelet and cardiac function using subtype-selective knockout mice. Cell Signal 19: 1765-1771, 2007.

26. Saito Y, Kondo H and Hojo Y: Granzyme B as a novel factor involved in cardiovascular diseases. J Cardiol 57: 141-147, 2011.

27. Stout-Delgado HW, Getachew Y, Rogers TE, Miller BC and Thiele DL: The role of serpinb9/serine protease inhibitor 6 in preventing granzyme B-dependent hepatotoxicity. Hepatology 46: 1530-1540, 2007.

28. Bird CH, Blink EJ, Hirst CE, Buzza MS, Steele PM, Sun J, Jans DA and Bird PI: Nucleocytoplasmic distribution of the ovalbumin serpin PI-9 requires a nonconventional nuclear import pathway and the export factor Crm1. Mol Cell Biol 21: 5396-5407, 2001.

29. Fayad W, Rickardson L, Haglund C, Olofsson MH, D'Arcy P, Larsson R, Linder S and Fryknäs M: Identification of agents that induce apoptosis of multicellular tumour spheroids: Enrichment for mitotic inhibitors with hydrophobic properties. Chem Biol Drug Des 78: 547-557, 2011.

30. Kisselev AF, van der Linden WA and Overkleeft HS: Proteasome inhibitors: An expanding army attacking a unique target. Chem Biol 19: 99-115, 2012.

31. Zheng Q, Su H, Tian Z and Wang X: Proteasome malfunction activates macroautophagy in the heart. Am J Cardiovasc Dis 1: 214-226, 2011.

32. Stangl K, Günther C, Frank T, Lorenz M, Meiners S, Röpke T, Stelter L, Moobed M, Baumann G, Kloetzel PM and Stangl V: Inhibition of the ubiquitin-proteasome pathway induces differential heat-shock protein response in cardiomyocytes and renders early cardiac protection. Biochem Biophys Res Commun 291: $542-549,2002$. 